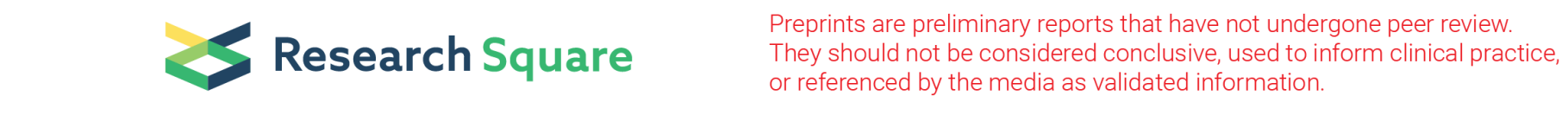

\title{
Effects of the ABCG2 C421A Polymorphism on Plasma Gefitinib Concentration
}

Sho Sakamoto ( $\sim$ ssakamoto@med.akita-u.ac.jp )

Department of Respiratory Medicine, Akita University Graduate Scool of Medicine JAPAN

\section{Research article}

Keywords: Non-small cell lung cancer; EGFR mutation; gefitinib

Posted Date: July 5th, 2019

DOI: https://doi.org/10.21203/rs.2.10916/v1

License: (c) (i) This work is licensed under a Creative Commons Attribution 4.0 International License. Read Full License 


\section{Abstract}

Background In patients with epidermal growth factor (EGFR) exon 21 L858R mutation-positive non-small cell lung cancer (NSCLC) treated with gefitinib, we found that a lower plasma gefitinib concentration was associated with shorter progression-free survival (PFS). ATP-binding castle protein G2 (ABCG2) is considered to inhibit the activities of certain gefitinib transporters, which affects drug pharmacokinetics. The C421A polymorphism affects the function and expression of ABCG2 on cell membrane. Previous studies showed that proton pump inhibitors (PPIs) inhibit gefitinib absorption, as well as the function of ABCG2. We evaluated the plasma concentrations of gefitinib in patients with and without the ABCG2 C421A polymorphism, who were or were not taking PPIs. Methods A total of 61 patients with advanced EGFR-positive NSCLC were enrolled in this study. They were treated with gefitinib at a dose of $250 \mathrm{mg}$ per day. Plasma gefitinib concentration and ABCG2 C421A status were determined after 2 weeks. The patients were divided into CC- and CA/AA genotype groups. We compared the trough and peak gefitinib levels, and the area under the curve (AUC) value for 24-hour gefitinib concentration. We also compared these parameters among four groups distinguished according to the presence/absence of the polymorphism and PPI use. Results The mean trough gefitinib level, and the AUC value for 24-hour gefitinib concentration, were significantly lower in the CA/AA group compared to the CC group (mean trough level: $333.2 \pm 188.7$ vs. $454.5 \pm 206.3 \mathrm{ng} / \mathrm{mL}, \mathrm{P}=0.021$; AUC: 9,949.9 \pm 5,058.9 vs. 13,085.4 $\pm 5,075.3 \mathrm{ng}$ $\mathrm{h} / \mathrm{mL}, \mathrm{P}=0.034$ ). Among patients taking PPIs, the mean trough gefitinib level was significantly lower in the CA/AA group than the CC group (220.1 \pm 112.6 vs. $340.5 \pm 98.3 \mathrm{ng} / \mathrm{mL}, \mathrm{P}=0.033)$. Conclusions The CA/AA-type of ABCG2 C421A polymorphism may be associated with lower gefitinib plasma concentration.

\section{Background}

Gefitinib is a first-generation oral epidermal growth factor receptor (EGFR) tyrosine kinase inhibitor (TKI). This drug has been used clinically for treatment of patients with non-small cell lung cancer (NSCLC) harboring EGFR mutations, and has been shown to possess strong antitumor activity. Gefitinib is clinically useful in elderly patients, and in those with poor World Health Organization (WHO) performance status (PS) $(1,2)$; thus, it remains as one of the standard therapies for NSCLC harboring EGFR mutations.

Gefitinib therapy shows large variability in its antitumor efficacy among patients, and it is important to identify the factors responsible for this difference. Resistance mutations, such as T790M, are considered to be the main causes of gefitinib resistance. However, several other factors have been suggested to influence the efficacy of gefitinib, including body surface area (BSA), smoking history, and treatment with proton pump inhibitors (PPIs) $(3-5)$.

It is important to achieve a good understanding of the pharmacokinetics of drugs to predict their clinical outcomes. Adverse effects and clinical efficacy are closely associated with the degree of drug exposure $(6,7)$. Several groups have examined the association of plasma gefitinib concentration with its efficacy and the incidence rate of adverse effects; for example, lower plasma concentrations were reported to be associated with less efficacious gefitinib therapy $(8,9)$. Therefore, it is important to maintain high plasma concentrations of this agent, and to identify the factors affecting its pharmacokinetics.

Several factors are known to affect the pharmacokinetics of gefitinib. The stomach $\mathrm{pH}$ affects the absorption of gefitinib into the blood (10). The liver is the main organ associated with the activities of metabolic enzymes and transporters involved in the metabolism and excretion of gefitinib (11-14). However, there have been no clinical studies regarding the associations between these enzymes and the transporters and pharmacokinetics of gefitinib.

ATP-binding castle protein $\mathrm{G} 2$ (ABCG2) is one of the most intensively studied transporters present on cell membranes in the liver, urinary tract, intestinal duct, and tumor cells (15). We focused on the role of ABCG2 in the efflux and metabolism of gefitinib in the liver $(16,17)$. ABCG2 was shown to affect the uptake of gefitinib in cells in vitro $(18)$. Higher levels of 
ABCG2 expression are believed to be related to lower concentrations of gefitinib, while the function of ABCG2 is known to be inhibited by several PPIs $(19,20)$. Interindividual differences in the activity of ABCG2, according to genetic factors and use of other drugs, may be related to gefitinib plasma concentrations.

ABCG2 has a number of single nucleotide polymorphisms (SNPs), one of which, C421A, is known to reduce the level of ABCG2 expression $(21,22)$. The C421A allele frequency is very high in Asian populations $(23,24)$. The effects of the ABCG2 C421A gene polymorphism on the pharmacokinetics of gefitinib have not been elucidated.

This study was performed to test the hypothesis that the plasma gefitinib concentration differs according to ABCG2 C421A status and use of PPIs.

\section{Methods}

\section{Patients and protocols}

A total of 61 patients with advanced EGFR-positive NSCLC were enrolled in this study, between May 2010 and May 2017, at Akita University Hospital, Akita, Japan. All patients had a WHO PS of 0-1, and a single, or no, cytotoxic chemotherapy regimen had been applied previously. Gefitinib was administrated at a dose of $250 \mathrm{mg}$ once a day until disease progression. On day 14 after commencement of treatment, plasma samples were obtained just before, and 1, 2, 4, 6, 8, 12, and 24 hours after, administration of $250 \mathrm{mg}$ of gefitinib. We determined ABCG2 C421A gene polymorphism status by analyzing the blood samples. The 61 patients were divided into two groups according to ABCG2 C421A genotype, i.e., wild-type (CC) or variant type (CA/AA), and we compared the trough and peak gefitinib levels, and area under the curve (AUC) value for the 24-hour plasma gefitinib concentration, between the genotype groups. We also compared these parameters between patients with and without PPI treatment (Figure 1).Finally, gefitinib concentrations were compared among four groups of patients distinguished according to ABCG2 C421A polymorphism and PPI use status.

This study was approved by the Institutional Review Board of Akita University Hospital, and all patients provided written informed consent prior to enrollment.

\section{Analysis of plasma gefitinib concentration}

Plasma gefitinib concentrations were measured by high-performance liquid chromatography (HPLC) with ultraviolet detection. Following the addition of erlotinib $(25 \mathrm{ng} / 10 \mathrm{~mL}$ methanol) to $100-\mathrm{mL}$ plasma samples as an internal standard, the samples were diluted with $900 \mathrm{~mL}$ of water. This mixture was then applied to an Oasis HLB solid-phase extraction cartridge that had been activated with methanol and water $(1.0 \mathrm{~mL}$ each). The cartridge was then washed with $1.0 \mathrm{~mL}$ of water and $1.0 \mathrm{~mL}$ of $40 \%$ methanol in water, and eluted with $1.0 \mathrm{~mL}$ of $100 \%$ methanol. Eluates were dried by vortex-vacuum evaporation at $60^{\circ} \mathrm{C}$ using a rotary evaporator (CVE-2AS; AS-ONE, Osaka, Japan). The resulting residue was then dissolved in $20 \mathrm{~mL}$ of methanol and $20 \mathrm{~mL}$ of mobile phase additives. Then, $20-\mathrm{mL}$ aliquots of the samples were processed by HPLC. The mobile phase was $0.5 \% \mathrm{KH}_{2} \mathrm{PO}_{4}(\mathrm{pH} 3.5)$ acetonitrile methanol (55:25:20, v/v/v). The flow rate was $0.5 \mathrm{~mL} / \mathrm{min}$, and detection was carried out at $250 \mathrm{~nm}$. The coefficients of variation for intra- and interday assays were $<8.4 \%$, and the accuracy of intra- and interday assays was within $3.1 \%$. The limit of quantification for gefitinib was $10 \mathrm{ng} / \mathrm{mL}$.

We measured the concentrations of gefitinib in plasma samples at eight time points (just before and $1,2,4,6,8,12$, and 24 hours after gefitinib administration), and the AUC value for 24-hour plasma gefitinib concentration was also calculated. 
DNA was extracted from peripheral blood samples using a QIAamp Blood Mini Kit (Qiagen, Tokyo, Japan) and stored at $-80^{\circ} \mathrm{C}$ until analysis. The ABCG 2 C421A polymorphism was genotyped by long polymerase chain reaction (PCR) analysis and the PCR-restriction fragment length polymorphism (RFLP) method, as described previously (25). The results of the PCR-RFLP analysis were confirmed using a fully automated SNP detection system (Prototype i-Densy ${ }^{\text {Tw}}$; Arkray Inc., Kyoto, Japan). The frequencies of all loci analyzed were in Hardy-Weinberg equilibrium.

\section{Statistical analysis}

Plasma gefitinib concentrations were compared using the two-sample $t$ test in each group, or the Mann-Whitney $U$ test if the data were non-parametric. For comparison of plasma gefitinib concentrations among four groups, Tukey's honestly significant difference test was applied if one-way ANOVA indicated significance. The $P$-values were two-sided, and $P<0.05$ was taken to indicate significance. All statistical analyses were performed using SPSS for Windows software (ver. 11.0; SPSS Inc., Chicago, IL, USA).

\section{Results}

\section{Patient characteristics}

There were no significant differences between the two genotype groups in terms of age, sex, body weight, or BSA; in levels of aspartate transaminase, alanine transaminase, serum albumin, or total bilirubin; or in clinical stage or EGFR mutations (Table 1). Regarding PPI use, eight patients were using lansoprazole, five were using esomeprazole, three were using rabeprazole, and one was using omeprazole.

\section{Plasma concentrations}

The trough gefitinib levels could be measured in 61 patients, but we could not measure the peak gefitinib levels, or AUC value for 24-hour gefitinib concentration, in 11 patients due to insufficient blood samples. Thus, we analyzed these latter two parameters in 50 patients (Figure 2).

Significant differences were found in the trough gefitinib levels and AUC value for 24-hour gefitinib concentration between the ABCG2 C421A genotype groups (Figure 3); the mean trough level for the CA/AA group was $333.2 \pm 188.7 \mathrm{ng} / \mathrm{mL}$ and that of the CC group was $454.5 \pm 206.3 \mathrm{ng} / \mathrm{mL}(P=0.021)$; the respective AUC values were $9,949.9 \pm 5,058.9 \mathrm{ng} \square \mathrm{h} / \mathrm{mL}$ and $13,085.4 \pm 5,075.3 \mathrm{ng} \square \mathrm{h} / \mathrm{mL}(P=0.034)$. There was no difference in peak gefitinib level between the CA/AA and CC groups.

Comparison of the trough gefitinib levels between the four groups distinguished according to SNPs and PPI use (Figure 4) showed a significant difference between group 1 (CC without PPIs) and group 4 (CA/AA with PPIs) (495.5 \pm 220.6 $\mathrm{ng} / \mathrm{mL}$ vs. $220.1 \pm 112.6 \mathrm{ng} / \mathrm{mL}$, respectively, $P=0.000$ ); between group 2 (CA/AA without PPIs) and group 4 (380.8 \pm $196.0 \mathrm{ng} / \mathrm{mL}$ vs. $220.1 \pm 112.6 \mathrm{ng} / \mathrm{mL}$, respectively, $P=0.041$ ); and between group 3 (CC with PPIs) and group 4 (340.5 \pm $98.3 \mathrm{ng} / \mathrm{mL}$ vs. $220.1 \pm 112.6 \mathrm{ng} / \mathrm{mL}$, respectively, $P=0.033$ ). The peak plasma gefitinib level and AUC value for 24 -hour gefitinib concentration showed similar trends, but the group differences were not significant (data not shown).

\section{Discussion}


This is the first report regarding the relationships among ABCG2 C421A polymorphism, usage of PPIs, and plasma concentrations of gefitinib. Comparison of patients divided into two groups according to ABCG2 C421A genotype status showed that the variant polymorphism was associated with lower plasma concentrations of gefitinib. Comparison of the trough gefitinib levels among the four groups distinguished on the basis of SNPs and PPI use showed that the ABCG2 C421 A polymorphism and usage of PPIs were related to lower plasma concentrations of gefitinib.

The EGFR-TKIs include gefitinib, erlotinib, afatinib, and osimertinib, which are standard clinical therapies selected according to the patient's type of mutation, age, WHO PS, and comorbidities (26). Gefitinib is used in elderly patients, and in those with a poor PS $(1,2)$. Gefitinib is known to have a better safety profile, i.e., is associated with fewer adverse effects, than other TKIs $(1,2)$. In our previous study, we showed that a lower trough level of gefitinib was associated with shorter progression-free survival (PFS) in patients with exon 21 L858R mutations (27). It has also been suggested that plasma gefitinib concentration may be related to prognosis $(8,9)$. Several factors have been reported to affect the absorption, distribution, metabolism, and excretion of gefitinib $(10,12,28,29)$. Therefore, it is important to determine the factors that are most strongly associated with plasma gefitinib concentrations.

In a previous study, the plasma gefitinib concentration reached a steady-state level by around day 7 (30). Elsewhere, continuous administration of gefitinib typically led to 2-8-fold accumulation in plasma concentration, and the maximum concentration of gefitinib increased proportionally (12). Gefitinib in blood is considered to be taken up into cells through certain transporters expressed on the cell membrane, metabolized by cytochrome P450 (CYP) enzymes in liver cells, and excreted in bile via as-yet unknown transporters. The bioavailability of gefitinib was shown to be reduced by $47 \%$ by sustained elevation of gastric $\mathrm{pH}$ (10). PPIs are known to reduce the plasma gefitinib concentration (28). A high rate of gefitinib clearance by CYPs has been reported, which is associated with lower therapeutic efficacy (12). The CYP2D6 gene polymorphism was reported to affect the plasma gefitinib concentration by increasing the efficiency of metabolism (31). Plasma gefitinib is excreted via bile into feces, and much higher levels are found in feces than in urine (30). Therefore, the liver is the main organ involved in both the metabolism and excretion of gefitinib. Horak et al. reported that liver dysfunction influenced the pharmacokinetics of gefitinib (29).

Drug transporters also play significant roles in the pharmacokinetics of drugs. ABCG2, one of the most well-studied membrane transporters, has been shown to be expressed in the apical cellular membranes of healthy organs, including the liver, intestine, and kidney, as well as in tumors. ABCG2 is thought to play an important role in removing toxic substances from cells (15). Gefitinib had been shown to be a substrate for ABCG2, along with rosuvastatin, methotrexate, and sulfasalazine (19). Galetti et al. reported the results of several in vitro studies on the function of ABCG2 (18), and suggested that gefitinib is not a substrate of ABCG2 on the basis of a clear dissociation in the intracellular accumulation and efflux of gefitinib between groups treated and not treated with the ABCG2 inhibitor, fumitremorgin C. Moreover, they noted increased uptake of gefitinib in NSCLC with use of an ABCG2-silencing RNA (siRNA) technique. They also reported decreased intracellular accumulation of gefitinib in cells overexpressing ABCG2, and that the expression levels of several transporters related to gefitinib uptake were decreased in ABCG2-overexpressing cells. These results suggested that gefitinib is not a substrate of $A B C G 2$, and that $A B C G 2$ affects the intracellular accumulation of gefitinib by modulating its uptake rather than its efflux. Therefore, $A B C G 2$ is a protein associated with the metabolism and excretion of gefitinib. ABCG2 is assumed to increase the plasma concentration of gefitinib and negatively regulate uptake into liver cells. In the present study, we focused on the effects of the ABCG2 polymorphism on the pharmacokinetics of gefitinib.

Resequencing of ABCG2 revealed a number of allelic variants that may affect the activity of the gene product in vivo (15). $A B C G 2$ C421A is a SNP in exon 5 of the ABCG2 gene. The transversion associated with this SNP results in transformation of lysine to glutamine at codon 141 (Q141K) (22), associated with altered substrate specificity and protein function (32). Franke et al. suggested that ABCG2 SNP analysis may be useful for estimating systemic ABCG2 (15). Cusatis et al. reported that ABCG2 C421A altered the pharmacokinetics of other drugs in addition to gefitinib (33). The wild-type (CC) 
ABCG2 C421A polymorphism is known to be associated with higher concentrations of gefitinib than the variant type (CA/AA). Other SNPs have also been identified, but it remains unclear whether they are associated with the expression and function of the ABCG2 protein (34).

In previous reports regarding gefitinib therapy and ABCG2 C421A, it was unclear whether the polymorphisms were associated with clinical efficacy. Some previous studies showed that gefitinib is associated with a higher frequency of adverse effects in wild-type versus variant-type ABCG2 C421A (35-37). Meanwhile, Cusatis et al. reported that patients with reduced $A B C G 2$ activity due to genetic variants are at increased risk of diarrhea on gefitinib treatment (35). Moreover, Ma et al. reported that patients carrying the A allele of C421A tended to have a higher risk of hepatotoxicity on receipt of gefitinib therapy (37). These observations may be related to the higher concentrations of ABCG2 in interstitial or liver cells, and to the cell injuries associated with reduced ABCG2 activity, which inhibited gefitinib transport into cells. Kobayashi reported a difference in the plasma concentration of gefitinib by ABCG2 genotype (38). Our results showed a similar trend, in that the gefitinib concentration was lower in the CA/AA-versus CC-type ABCG2 group.

In this study, the relationship between ABCG2 genotype and PPI use was shown to be important in plasma gefitinib concentrations. The differences in gefitinib trough levels between groups distinguished according to ABCG2 genotype and PPI use were considered to be due to an interaction effect, where PPI use may influence plasma concentrations of gefitinib by inhibiting the activities of ABCG2. The differences in gefitinib plasma concentrations between groups 1 (CC without PPIs) and 3 (CC with PPIs), and between groups 2 (CA/AA without PPIs) and 4 (CA/AA with PPIs), were significant. These results suggested that PPIs may be more directly associated with the plasma concentration of gefitinib than ABCG2 genotype. Considering the significant difference in trough gefitinib level between groups 2 and 4, PPI use should be avoided in patients with the variant-type ABCG2 polymorphism to maintain therapeutic plasma concentrations of gefitinib.

Several drugs are known to inhibit the function of ABCG2, including PPIs and cyclosporine (19). Suzuki identified coadministration of several PPIs as a risk factor for delayed elimination of gefitinib, as well as for renal and liver dysfunction (20). All four PPIs used in their study (omeprazole, rabeprazole, lansoprazole, pantoprazole) inhibited ABCG2mediated transport of methotrexate (20). However, in previous studies on the relationship between ABCG2 and PPIs, pantoprazole and rabeprazole were the only two agents that met the criteria for clinical inhibitors (19). Therefore, although PPIs play a role in inhibiting the function of ABCG2, they may not have strong effects on the pharmacokinetics.

This study had some limitations. First, it was unclear how the differences among the various PPIs may have affected the results. Thus, investigations using a single PPI may be preferable. Second, the gefitinib concentrations may have been biased in one patient in group 3 due to with pulverization and solution of gefitinib tablet via a nasal gastric tube. This may have affected the absorption and, subsequently, concentration of gefitinib in plasma. Third, the sample size was relatively small and we could not measure the peak gefitinib levels, or AUC value for 24-hour gefitinib concentration, in 11 patients due to insufficient blood samples. Further studies including larger samples are needed to confirm the association between C421A polymorphism and plasma concentrations of gefitinib.

\section{Conclusions}

ABCG2 C421A gene polymorphism may affect the plasma concentration of gefitinib. In patients with the CA or AA ABCG2 C421A genotype, PPIs may be associated with lower plasma gefitinib concentrations.

\section{References}

1 Maemondo M, Minegishi Y, Inoue A, Kobayashi K, Harada M, Okinaga S, Morikawa N, Oizumi S, Tanaka T, Isobe H, Kudoh S, Hagiwara K, Nukiwa T, and Gemma A. First-line gefitinib in patients aged 75 or older with advanced non-small 
cell lung cancer harboring epidermal growth factor receptor mutations: NEJ 003 study. J Thorac Oncol. 2012 Sep;7(9):1417-22.

2 Inoue A, Kobayashi K, Usui K, Maemondo M, Okinaga S, Mikami I, Ando M, Yamazaki K, Saijo Y, Gemma A, Miyazawa H, Tanaka T, Ikebuchi K, Nukiwa T, Morita S, Hagiwara K; North East Japan Gefitinib Study Group. First-line gefitinib for patients with advanced non-small-cell lung cancer harboring epidermal growth factor receptor mutations without indication for chemotherapy. J Clin Oncol. 2009 Mar 20;27(9):1394-400. doi: 10.1200/JC0.2008.18.7658. Epub 2009 Feb 17.

3 Eiki Ichihara, Katsuyuki Hotta, Nagio Takigawa, Kenichiro Kudo, Yuka Kato,

Yoshihiro Honda, Hiromi Hayakawa, Daisuke Minami, Akiko Sato, Masahiro Tabata, Mitsune Tanimoto, Katsuyuki Kiura: Impact of physical size on gefitinib efficacy in patients with non-small cell lung cancer harboring EGFR mutations. Lung Cancer 81 (2013) 435- 439

4 Igawa S. - Sasaki J. · Otani S. - Ishihara M. · Takakura A. · Katagiri M. · Masuda N.: Impact of Smoking History on the Efficacy of Gefitinib in Patients with Non-Small Cell Lung Cancer Harboring Activating Epidermal Growth Factor Receptor Mutations. Oncology 2015; 89:275-280

5 KUNIHIKO MIYAZAKI, SHINYA SATO, TAKAHIDE KODAMA, TOMOHIRO TAMURA, KATSUNORI KAGOHASHI, HIROAKI SATOH and NOBUYUKI HIZAWA, Effect of acid suppressants on the efficacy of tyrosine kinase inhibitors in patients with epidermal growth factor receptor-mutated non-small-cell lung cancer. MOLECULAR AND CLINICAL ONCOLOGY 4: 873-877, 2016

6 Shigehiro Yagishita and Akinobu Hamada. Clinical Pharmacology of EGFR/Met Inhibitors in Non-Small Cell Lung Cancer. Current Drug Targets, 2014, 15, 1263-1272

7 Samir D. Undevia, Gonzalo Gomez-Abuin and Mark J. Ratain. Pharmacokinetic Variability Of Anticancer Agents: Nature, VOLUME 5, JUNE 2005, 457-458

8 Shuang Xin, Yuanyuan Zhao, Xueding Wang, Yan Huang, Jing Zhang, Ying Guo, Jiali Li, Hongliang Li, Yuxiang Ma, Lingyan Chen, Zhihuang Hu, Min Huang, and Li Zhangb. The Dissociation of Gefitinib Trough Concentration and Clinical Outcome in NSCLC Patients with EGFR Sensitive Mutations. Scientific Reports | 5:12675 | DOI: 10.1038/srep12675 
9 Yuan-Yuan Zhao, Su Li, Yang Zhang, Hong-Yun Zhao, Hai Liao, Ying Guo, Yan-Xia Shi , Wei Jiang, Cong Xue, Li Zhang: The relationship between drug exposure and clinical outcomes of non-small cell lung cancer patients treated with gefitinib. Med Oncol (2011) 28:697-702

10 European Medicines Agency. Iressa (gefitinib) Assessment Report, July 2009.

http://www.ema.europa.eu/docs/en_GB/document_library/EPAR_-

_Public_assessment_report/human/001016/WC500036361.pdf. Feb 20, 2014

11 Jie S, John S.M, Di B, Guohua A. Enzyme-and transporter- Mediated Drug Interactions with Small Molecule Tyrosine Kinase Inhibitors. Journal of pharmaceutical sciences 103: 3810-3833, 2014

12 Can Zhao, Shu-Yan Han and Ping-Ping Li. Pharmacokinetics of Gefitinib: Roles of Drug Metabolizing Enzymes and Transporters. Current Drug Delivery, 2017, 14, 282-288

13 McKillop, AD McCormick, A Millar, GS Miles, PJ Phillips and M Hutchison: Cytochrome P450-dependent metabolism of gefitinib. Xenobiotica, Jan 2005, 35(1): 39-50

14 McKillop, AD McCormick, A Millar, GS Miles, PJ Phillips, KJ Pickup, N Bushby and M Hutchison: In vitro metabolism of gefitinib in human liver microsomes. Xenobiotica, Nov/ Dec 2004, vol 34, No 11/12, 983-1000

15 R.M. Franke, E.R. Gardner and Sparreboom: Pharmacogenetics of Drug Transporters. Current Pharmaceutical Design, $2010,16,220-230$

16 Li J, Cusatis G, Brahmer J, Sparreboom A, Robey RW, Bates SE, Hidalgo M, Baker SD: Association of variant ABCG2 and the pharmacokinetics of epidermal growth factor receptor tyrosine kinase inhibitors in cancer patients. Cancer Biol Ther. 2007 Mar;6(3):432-8. Epub 2007 Mar 29.

17 Wei Mo, Jian-Ting Zhang: Human ABCG2: structure, function, and its role in multidrug resistance. Int J Biochem Mol Biol 2012;3(1):1-27

18 Maricla Galetti, Pier Giorgio Petronini, Claudia Fumarola, Daniele Cretella, Silvia La Monica, Mara Bonelli, Andrea Cavazzoni, Francesca Saccani, Cristina Caffarra, Roberta Andreoli , Antonio Mutti, Marcello Tiseo, Andrea Ardizzoni, Roberta R. Alfieri: Effect of ABCG2/BCRP Expression on Efflux and Uptake of Gefitinib in NSCLC Cell Lines. PLOS ONE I DOI:10.1371/journal.pone.0141795 November 4, 2015 
19 Caroline A. Lee, Meeghan A. O'Connor, Tasha K. Ritchie, Aleksandra Galetin, Jack A. Cook, Isabelle RagueneauMajlessi, Harma Ellens, Bo Feng, Mitchell E. Taub, Mary F. Paine, Joseph W. Polli, Joseph A. Ware, and Maciej J. ZamekGliszczynski: Breast Cancer Resistance Protein (ABCG2) in Clinical Pharmacokinetics and Drug Interactions: Practical Recommendations for Clinical Victim and Perpetrator Drug-Drug Interaction Study Design. Drug Metab Dispos. 2015 Apr;43(4):490-509. doi: 10.1124/dmd.114.062174. Epub 2015 Jan 13.

20 Kunihiro Suzuki, Kosuke Doki, Masato Homma, Hirofumi Tamaki, Satoko Hori, Hisakazu Ohtani, Yasufumi Sawada \& Yukinao Kohda: Co-administration of proton

pump inhibitors delays elimination of plasma methotrexate in high-dose methotrexate therapy. Br J Clin Pharmacol / 67:1 / 44-49

21 Daisuke Kobayashi, Ichiro leiri, Takeshi Hirota, Hiroshi Takane, Shinji Maegawa, Junzo Kigawa, Hiroshi Suzuki, Eiji Nanba, Mitsuo Oshimura, Naoki Terakawa, Kenji Otsubo, Kazunori Mine and Yuichi Sugiyama: Functional Assessment Of ABCG2 (BCRP) Gene Polymorphisms To Protein Expression In Human Placenta. DRUG METABOLISM AND DISPOSITION Vol. 33, No. 1, 33:94-101, 2005

22 Imai Y, Nakane M, Kage K, Tsukahara S, Ishikawa E, Tsuruo T, Miki Y, Sugimoto Y. C421A polymorphism in the human breast cancer resistance protein gene is associated with low expression of Q141K protein and low-level drug resistance. Mol Cancer Ther. 2002; 1(8):611-616.

23 Maeda K, Sugiyama Y. Impact of genetic polymorphisms of transporters on the pharmacokinetic, pharmacodynamic and toxicological properties of anionic drugs. Drug Metab Pharmacokinet. 2008; 23(4):223-235. doi:

10.2133/dmpk.23.223.

24 Mizuarai S, Aozasa N, Kotani H. Single nucleotide polymorphisms result in impaired membrane localization and reduced atpase activity in multidrug transporter ABCG2. Int J Cancer. 2004; 109(2):238-246. doi: 10.1002/ijc.11669.

25 Kobayashi D, leiri I, Hirota T, Takane H, Maegawa S, Kigawa J, Suzuki H, Nanba E, Oshimura M, Terakawa N, Otsubo K, Mine K, Sugiyama Y. Functional assessment of ABCG2 (BCRP) gene polymorphisms to protein expression in human placenta. Drug Metab Dispos. 2005; 33:94-101.

26 Oscar Juan and Sanjay Popat: Treatment choice in epidermal growth factor receptor mutation-positive non-small cell lung carcinoma: latest evidence and clinical implications. Ther Adv Med Oncol 2017, Vol. 9(3) 201-216 
27 Yuji Okuda, Kazuhiro Sato, Kazuhisa Sudo, Yukiyasu Hasegawa, Mariko Asano, Hajime Miura, Masahide Takeda, Masaaki Sano, Hiroyuki Watanabe, Hiroyuki Kobayashi, Takenori Niioka, Masatomo Miura, Hiroshi Ito. Low plasma concentration of gefitinib in patients with EGFR exon 21 L858R point mutations shortens progression-free survival. Cancer Chemother Pharmacol (2017) 79:1013-1020

28 Hayato Yokota, Kazuhiro Sato, Yuji Okuda, Hiroyuki Kobayashi, Masahide Takeda, Mariko Asano, Hiroshi Ito, Masatomo Miura: Effects of Histamine 2-receptor Antagonists and Proton Pump Inhibitors on the Pharmacokinetics of Gefitinib in Patients With Nonesmall-cell Lung Cancer. Clin Lung Cancer. 2017 Nov; 18 (6): e433-e439.

29 Jiri Horak, Jeff White, Adrian L. Harris, Mark Verrill, James Carmichael, Alison Holt, Mireille Cantarini, Merran Macpherson, Alan Swaisland, Helen Swaisland, Chris Twelves: The effect of different etiologies of hepatic impairment on the pharmacokinetics of gefitinib. Cancer Chemother Pharmacol (2011) 68:1485-1495

30 Swaisland HC1, Cantarini MV, Fuhr R, Holt A. , Exploring the relationship between expression of cytochrome P450 enzymes and gefitinib pharmacokinetics. Clin Pharmacokinet. 2006;45(6):633-44.

31 Cohen MH, Williams, G.A et al. United States food and drug administration drug approval summary: Gefitinib tablets. Clin Cancer Res, 2004, 10(4), 1212-1218

32 Gardner ER, Figg WD, Sarreboom A: Pharmacogenenomics of the Human ATP-Bindng Castle Transporrter ABCG2. Current Pharmacogenomics 2006; 4(4): 331-44

33 Cusatis G, Sparreboom A: Pharmacogenomic importance of ABCG2. Pharmacogenomics 2008, 9(8):1005-9.

34 Xueqin Chen, Dadong Chen, Shaoyu Yang, Ruobing Ma, Yuelong Pan, Xin Li and Shenglin Ma: Impact of ABCG2 polymorphisms on the clinical outcome of TKIs therapy in Chinese advanced non-small-cell lung cancer patients. Cancer Cell International (2015) 15:43

35 Cusatis G, Gregorc V, Li J, Spreafico A, Ingersoll RG, Verweij J, Ludovini V, Villa E, Hidalgo M, Sparreboom A, Baker SD: Pharmacogenetics of ABCG2 and adverse reactions to gefitinib. J Natl Cancer Inst. 2006 Dec 6; 98(23):1739-42.

36 Lina Tang, Chunling Zhang, Hairong He, Zhenyu Pan, Di Fan, Yinli He, Haisheng You, and Yuanjie Li: Associations between ABCG2 gene polymorphisms and gefitinib toxicity in non-small cell lung cancer: a meta-analysis. Onco Targets Ther. 2018; 11: 665-675. 
$37 \mathrm{Ma}$ Y, Xin S, Huang M, et al. Determinants of Gefitinib toxicity in advanced non-small cell lung cancer (NSCLC): a pharmacogenomic study of metabolic enzymes and transporters. Pharmacogenomics J. 2016; 17(4):325-330

38 Hiroyuki Kobayashi, Kazuhiro Sato, Takenori Niioka, Masahide Takeda, Yuji Okuda, Mariko Asano, Hiroshi Ito, Masatomo Miura: Effects of polymorphisms in CYP2D6 and ABC transporters and side effects induced by gefitinib on the pharmacokinetics of the gefitinib metabolite, O-desmethyl gefitinib. Med Oncol (2016) 33:57

\section{Tables}

Table 1. Patient characteristics

\begin{tabular}{|c|c|c|c|c|c|c|c|c|}
\hline & $\begin{array}{c}\text { Group } 1 \\
\text { (PPI-, CC) }\end{array}$ & $\begin{array}{l}\text { Group } 2 \\
\text { (PPI-, } \\
\text { CA/AA) }\end{array}$ & $\begin{array}{c}\text { Group } 3 \\
\text { (PPI+, CC) }\end{array}$ & $\begin{array}{l}\text { Group } 4 \\
\text { (PPI+, } \\
\text { CA/AA) }\end{array}$ & $\begin{array}{c}\mathrm{P}- \\
\text { value }\end{array}$ & $\begin{array}{l}\text { Group } 1+3 \\
\text { (CC) }\end{array}$ & $\begin{array}{c}\text { Group } 2+4 \\
\text { (CA/AA) }\end{array}$ & $\begin{array}{c}\mathrm{P}- \\
\text { value }\end{array}$ \\
\hline $\begin{array}{c}\text { Number of } \\
\text { subjects }\end{array}$ & 25 & 19 & 9 & 8 & & 34 & 27 & \\
\hline Age(y) & $69.6 \pm 6.69$ & $70.68 \pm 10.67$ & $73.56 \pm 7.48$ & $69.9 \pm 9.02$ & 0.251 & $70.6 \pm 7.02$ & $18.9 \pm 11.11$ & 0.474 \\
\hline $\begin{array}{l}\text { Sex } \\
\text { male } \\
\text { female }\end{array}$ & $\begin{array}{l}11 \\
14\end{array}$ & $\begin{array}{c}2 \\
17\end{array}$ & $\begin{array}{l}2 \\
7\end{array}$ & $\begin{array}{l}4 \\
4\end{array}$ & 0.101 & $\begin{array}{l}13 \\
21\end{array}$ & $\begin{array}{c}6 \\
21\end{array}$ & 0.092 \\
\hline $\mathrm{BW}(\mathrm{kg})$ & $54.3 \pm 9.04$ & $50.6 \pm 7.9$ & $48.2 \pm 9.48$ & $54.5 \pm 14.92$ & 0.317 & $52.7 \pm 9.41$ & $51.8 \pm 10.32$ & 0.72 \\
\hline $\mathrm{BSA}(\mathrm{m} \square)$ & $1.53 \pm 0.17$ & $1.45 \pm 0.11$ & $1.41 \pm 0.16$ & $1.53 \pm 0.26$ & 0.189 & $1.50 \pm 0.17$ & $1.48 \pm 0.17$ & 0.606 \\
\hline AST(IU/L) & $28.3 \pm 27.98$ & $47.1 \pm 115.65$ & $22.0 \pm 12.59$ & $20.2 \pm 6.36$ & 0.694 & $26.6 \pm 24.82$ & $39.1 \pm 97.09$ & 0.674 \\
\hline ALT(IU/L) & $35.2 \pm 55.55$ & $57.4 \pm 162.88$ & $27.8 \pm 34.92$ & $20.3 \pm 10.07$ & 0.781 & $33.3 \pm 50.51$ & $46.41 \pm 36.72$ & 0.606 \\
\hline $\mathrm{Alb}(\mathrm{g} / \mathrm{dL})$ & $3.8 \pm 0.42$ & $3.9 \pm 0.38$ & $3.5 \pm 0.52$ & $3.6 \pm 0.77$ & 0.144 & $3.78 \pm 0.43$ & $3.85 \pm 0.43$ & 0.594 \\
\hline T. Bil(mg/dL) & $0.63 \pm 0.27$ & $0.6 \pm 0.19$ & $0.53 \pm 0.15$ & $0.46 \pm 0.18$ & 0.202 & $0.6 \pm 0.56$ & $0.56 \pm 0.2$ & 0.443 \\
\hline $\begin{array}{c}\text { cStage } \\
\square\end{array}$ & $\begin{array}{c}3 \\
12\end{array}$ & $\begin{array}{c}2 \\
17\end{array}$ & $\begin{array}{l}1 \\
8\end{array}$ & $\begin{array}{l}0 \\
8\end{array}$ & 0.128 & $\begin{array}{c}4 \\
30\end{array}$ & $\begin{array}{c}2 \\
25\end{array}$ & 0.293 \\
\hline$\square$ & & & & & & & & \\
\hline $\begin{array}{c}\text { EGFR } \\
\text { mutation } \\
\text { L858R } \\
\text { del19 }\end{array}$ & $\begin{array}{c}15 \\
8 \\
2\end{array}$ & $\begin{array}{c}11 \\
7 \\
1\end{array}$ & $\begin{array}{l}5 \\
4 \\
0\end{array}$ & $\begin{array}{l}4 \\
4 \\
0\end{array}$ & 0.072 & $\begin{array}{c}20 \\
12 \\
2\end{array}$ & $\begin{array}{c}15 \\
11 \\
1\end{array}$ & 0.193 \\
\hline
\end{tabular}

\section{Declarations}

Acknowledgments 
This work was supported by the Department of Pharmacy of Akita University Hospital.

\section{Conflict of interest}

The authors have no conflicts of interests to disclose.

\section{Ethical approval}

All procedures in studies involving human participants were performed in accordance with the ethical standards of the institutional and national research committee and with the 1964 Declaration of Helsinki and its later amendments or comparable ethical standards.

Informed consent

Informed consent was obtained from all patients in this study.

\section{Figures}

\section{The gene-polymorphism of ABCG2 C421A}

\begin{tabular}{c|c|c|c|}
\cline { 2 - 4 } \multicolumn{1}{c|}{} & CC & CA/AA \\
\cline { 2 - 4 } \multicolumn{1}{c|}{} & - & $\begin{array}{c}\text { Group1 } \\
(\mathrm{n}=25)\end{array}$ & $\begin{array}{c}\text { Group2 } \\
(\mathrm{n}=19)\end{array}$ \\
\cline { 2 - 4 } & + & $\begin{array}{c}\text { Group3 } \\
(\mathrm{n}=9)\end{array}$ & $\begin{array}{c}\text { Group4 } \\
(\mathrm{n}=8)\end{array}$ \\
\hline
\end{tabular}

\section{Figure 1}

Classification of 61 patients with advanced non-small cell lung cancer (NSCLC) harboring epidermal growth factor (EGFR) mutations into four groups. 
A trough level

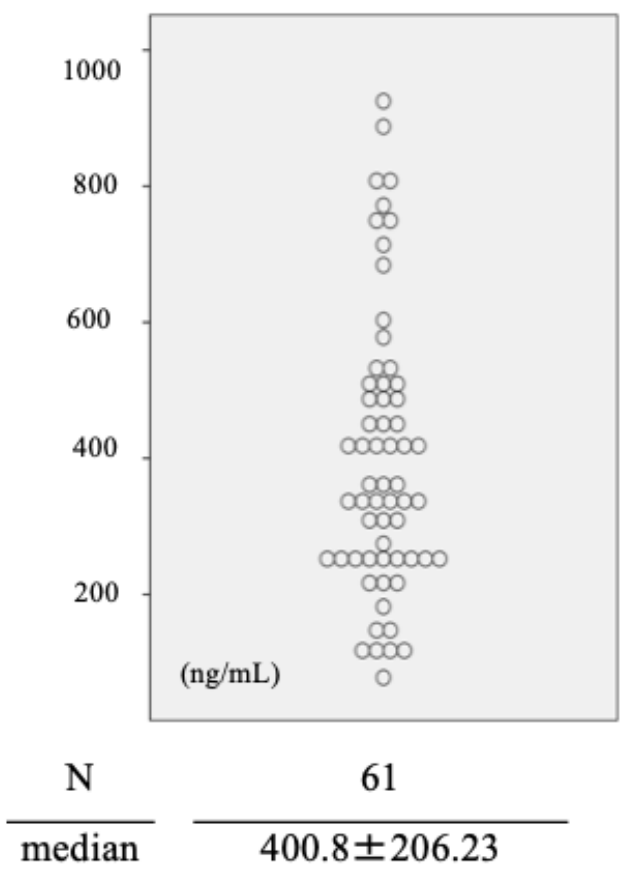

B peak level

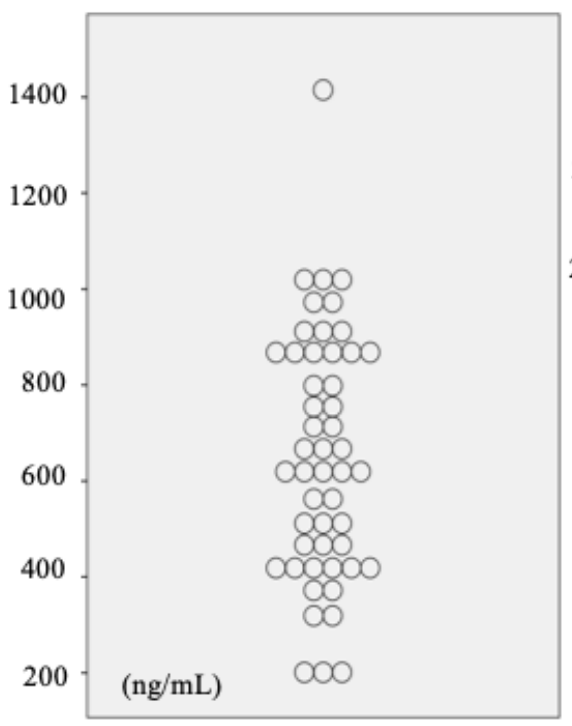

50

$651.4 \pm 257.53$
C AUC at 24-h

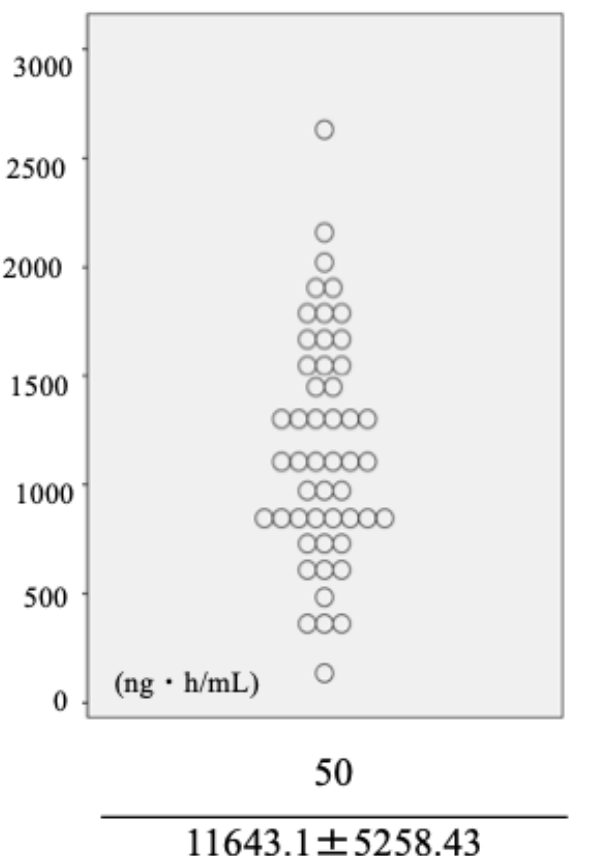

$11643.1 \pm 5258.43$

\section{Figure 2}

Distributions of trough and peak gefitinib levels, and the area under the curve (AUC) value for 24-hour plasma gefitinib.

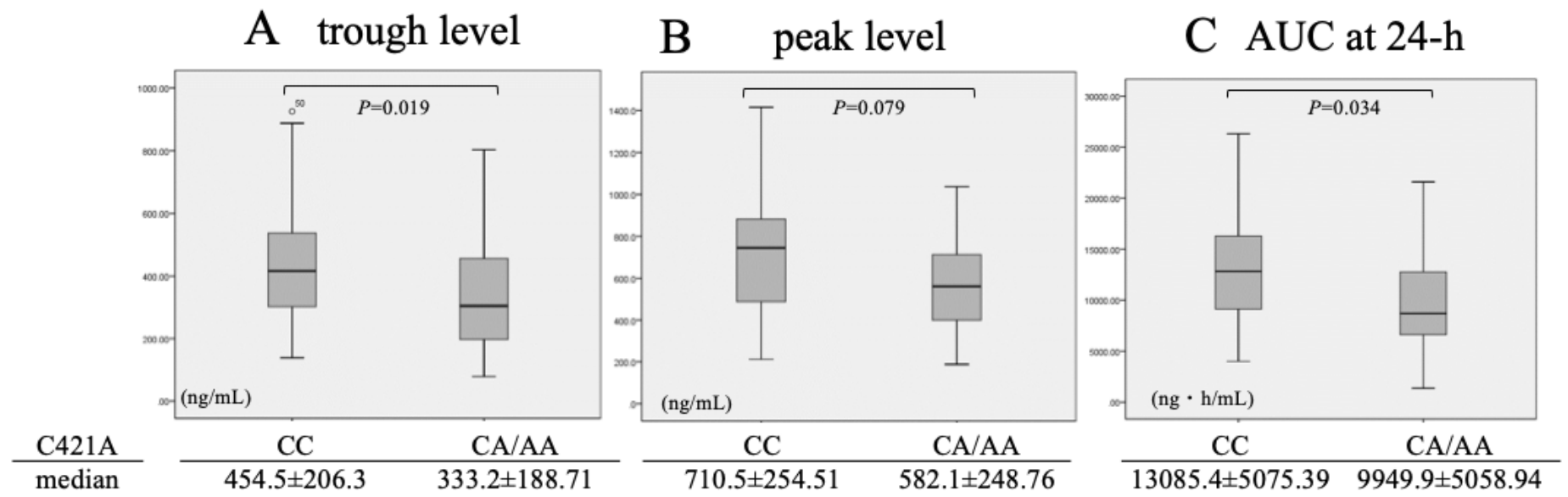

\section{Figure 3}

Comparison of plasma gefitinib concentrations according to ABCG2 C421A genotype status. 


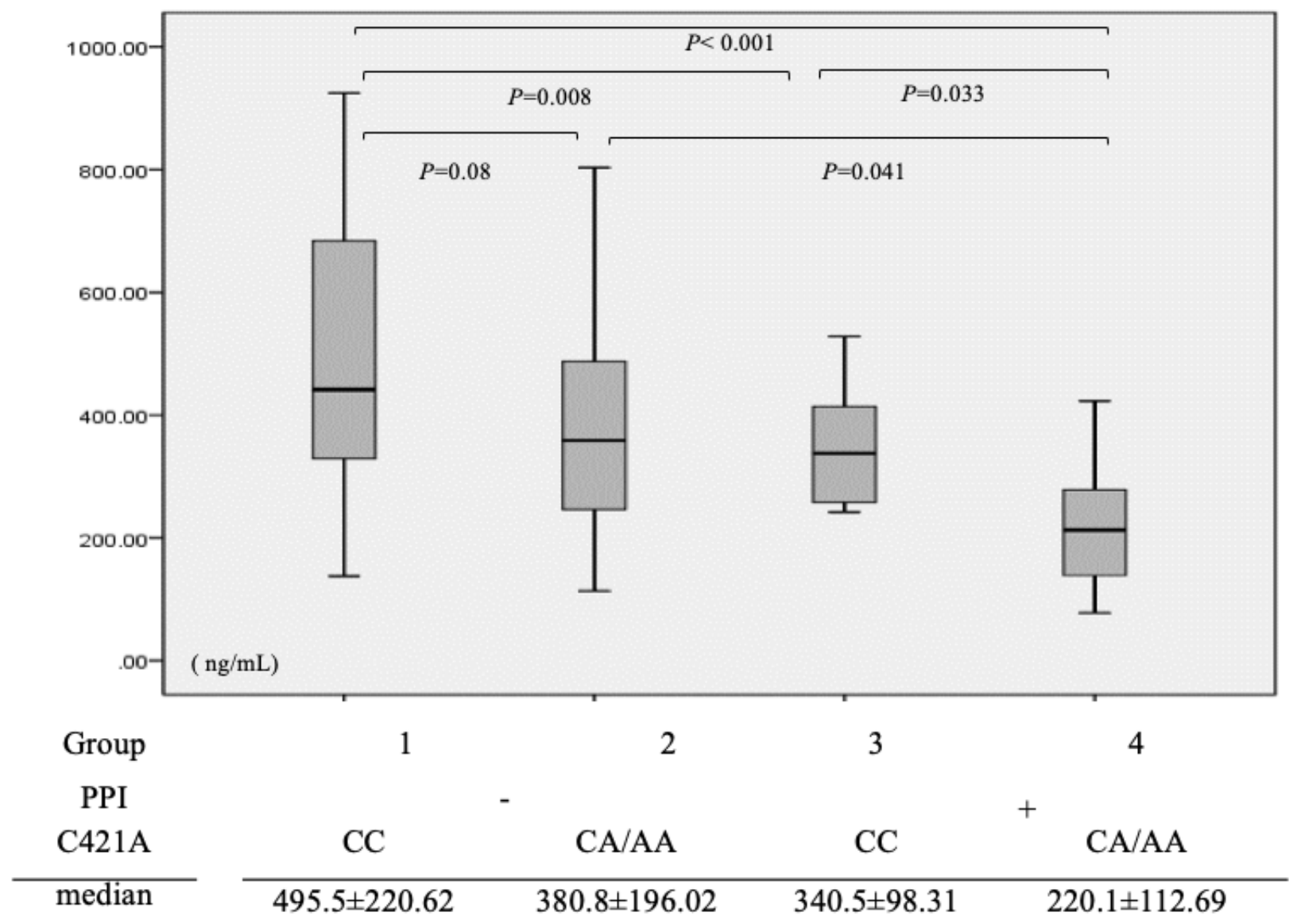

Figure 4

Comparison of trough gefitinib levels among four groups distinguished according to ABCG2 C421A genotype status and proton pump inhibitor (PPI) use. 